\title{
A Virtual Hosting Environment for Distributed Online Gaming
}

\author{
David Brossard and Juan Luis Prieto Martinez \\ BT Innovate, PP13D Orion Building, Adastral Park, \\ IP5 3RE Martlesham Heath, England \\ \{david.brossard, juanluis.prietomartinez\}@bt.com
}

\begin{abstract}
With enterprise boundaries becoming fuzzier, it's become clear that businesses need to share resources, expose services, and interact in many different ways. In order to achieve such a distribution in a dynamic, flexible, and secure way, we have designed and implemented a virtual hosting environment (VHE) which aims at integrating business services across enterprise boundaries and virtualising the ICT environment within which these services operate in order to exploit economies of scale for the businesses as well as achieve shorter concept-to-market time scales. To illustrate the relevance of the VHE, we have applied it to the online gaming world. Online gaming is an early adopter of distributed computing and more than $30 \%$ of gaming developer companies, being aware of the shift, are focusing on developing high performance platforms for the new online trend.
\end{abstract}

\section{Introduction}

Internet-based gaming offers challenging features such as interactivity with multiple players, latency requirements, high-performance servers for game execution, richness of the games, billing requirements, user privacy issues. But running a gaming infrastructure can be costly especially if under-used. It is critical that online gaming industries adopt technology that let them be more flexible and adaptive so they can securely address their customers' needs while delivering with the minimal amount of resources the quality the customer has requested. To address these issues, we have developed a Virtual Hosting Environment (VHE): it offers an advanced ICT environment that enables integrating business services to operate across enterprise boundaries over a virtualized ICT infrastructure. New reusable capabilities can be exposed as network-hosted services and be seamlessly introduced in the VHE to enhance its generic functionality or meet market-specific needs. This paper will describe the VHE applied to an online gaming scenario.

\section{Context}

The online gaming scenario contains four main actors: (1) the game player (GP) is the end consumer and is looking for a particular game title and game plan which suits his means; (2) the Online Game Platform Provider (OLG) identifies business opportunities, 
selects the game titles to offer, looks for game providers, forms the virtual organization (VO), and manages the match lifecycle for the selected game title; (3) the Games Server Provider (GA) offers game host environments, advertises game application services that meet certain QoS levels, and participates in VOs formed by OLG; (4) the Infrastructure Provider (ISP) enables VO lifecycle management, mediates GA discovery by the OLG, brokers agreements, facilitates the use of common capabilities (CC) offered by $3^{\text {rd }}$ party providers, and offers business service registries. Such CC include security capabilities (see $[1,2])$

There are four key areas in the gaming scenario: (1) the virtual organization management service (VOMS) is responsible for the definition \& establishment of secure federations between different partners. It maintains and publishes partners, services, policies, and roles. It drives partner selection based on service-level requirements; (2) the B2B gateway runs at each partner's site and interfaces between the VOMS and that partner's resources. It maintains its policies, list of services, and users. It enables resource virtualization and interfaces with the hosting environments (HE) as well as the value-added services (VAS); (3) the HE is a set of physical servers or virtual machines that can host the application to be virtualized. It offers interfaces to load applications, create instances, and capture QoS measurements; (4) VAS live in the cloud and can be used to support key areas such as security [1,2]. Other VAS include presence or telephony services e.g. VoIP.

Andago, a games company, wants to offer new games. It is looking for more games and hosting resources. It wants to delegate the hosting and other non-functional requirements to $3^{\text {rd }}$ parties. Andago wants to focus on their game portal and their users. Hence, Andago requests a VO creation: it will invite partners that will provide hosting as well as security, auditing, and SLA services. Andago invites suitable partners via the VOMS. Sunny and Saygah, two hosting enterprises will fulfill the GS role. Andago will fulfill the OLG role.

\section{Technical Approach}

Using the VOMS, Andago associates each role with policies which are sent to each partner's B2B gateway where they are refined and stored. Here, we decide to send access control policies for the GS and claims for the OLG. They will be used to configure the relevant infrastructure services. Once each partner accepts the VO invitation, Andago initiates the VO creation and orders are sent to each partner's B2B gateway to configure their infrastructure and to make their services available in the newly created VO. Each B2B gateway then looks for a suitable infrastructure profile to use to create its own view of the VO. Profiles point to different infrastructure services such as the security services in [2] or SLA services. In this scenario, we make use of a security token service (STS), an XML security gateway, and an authorization service. The XML security gateway is used to virtualize, contextualize, and expose each partner's selected service(s). At this point, each partner's B2B gateway governance layer creates a new federation at the corresponding STS and pushes it the partner business cards. The global UDDI is updated: each partner's services are made available for virtualization within the VO. At Sunny and Saygah, the advertised services are exposed - in this case the management web service for the hosted game (called a watcher). The infrastructure services are then configured with the relevant policies. 
Andago then consumes the services it wishes from Sunny and Saygah: Andago is after the EnemyTerritory game hosted at Sunny \& Saygah.

For the user, response time, availability, and gamer account security are critical. The player's interaction starts when he goes to Andago's website. He logs into Andago's web portal which manages users, games, and online communities. The user then chooses a game to play. Up to this point, all interactions take part solely between Andago and the end user. Once the player has selected a game, he chooses a match to join. This is where the VHE kicks in. A match is in fact a given specific VO with a virtualized exposed web service for one of the game servers (GS) selected e.g. Sunny. Once the player has selected a match, Andago starts the virtualization of its own client application, Agasy, which liaises between its platform and Sunny's virtualized watcher. At this stage, Andago is virtualising and exposing an instance of Agasy. The latter exchanges management \& monitoring messages with the Sunny watcher instance. When it calls the watcher instance, the request goes through the supporting infrastructure previously configured by the B2B gateway. The demonstrator will focus on the details of the interactions and how message exchanges are secured. In the following paragraph we have a closer look at which particular capabilities are used and for which purposes.

The key capabilities are the VOMS, the Federation Manager \& Identity Broker (STS), the Authorization Service (PDP), the XML Enforcement Gateway (PEP), and the SLA monitor (SLAm). The security infrastructure comes into play as follows: outbound, the message is intercepted by Andago's XML security gateway. There, the message is (1) checked and decorated with a token issued by Andago's STS; (2) checked for compliance against Andago's authorization rules at the PDP; (3) encrypted with a proof-of-possession (PoP) key embedded in the SAML token; and (4) sent to the virtual endpoint of the watcher instance at Sunny. Inbound, at Sunny's gateway, (1) the SAML token is extracted and sent to the STS; it (2) extracts the PoP and Agasy's identity claims; (3) the XML gateway decrypts the message and requests an authorization decision from the PDP based on the extracted claims; (4) the PDP returns a decision based on the claim, target, action, and environment information; (5) the gateway then forwards the request to the watcher instance. The demonstrator will also illustrate the use of an SLA system.

\section{Innovation}

Overall, the VHE drives and achieves total separation of concerns between functional and non-functional requirements. This in turn allows: (1) agile composition of services: offering capabilities as services is one step towards 'SaaS'; (2) optimized resource usage and reusable infrastructure; (3) increased flexibility: the VHE enables a total management \& adaptation of exposed services with zero downtime; (4) enriched user experience: with the SLA framework of the VHE in particular. Each capability has specific innovative features discussed in $[1,2]$.

\section{Business Impact}

We estimate up to $80 \%$ of enterprises anticipate the following benefits from the VHE: loosely coupled systems, service reuse, composite applications built by combining 
services, enabling response to changing market requirements and first-to-market competitive advantage, optimization of end-to-end processes, enabling a higher degree of automation, and compliance mechanisms. The VHE enables the customer to adopt a low-risk approach to SOA and increased return on investment. It provides common technologies that enable business processes to be added, changed, and removed easily. The VHE can support non-functional requirements e.g. QoS-based service publication, process-driven service composition; federated identity \& access control, and secure messaging.

The core market relevant to VHE is a segment of the Infrastructure Management services market. The opportunities for Communication and IT services companies in this market in Europe are predicted to reach $€ 61.3$ billion by 2009 . Services empower a wide variety of customers in multiple domains, e.g. Defence, Finance, or Media. The VHE offers a strategic advantage to Communications and IT Services companies when competing with SOA vendors. They increase their cost effectiveness, decrease concept-to-market timescales and create economies of scale.

Acknowledgments. The paper is the result of work from EU projects TrustCoM, BEinGRID, and GridTrust. In addition, components from EU project GRASP are used in the demonstration.

\section{References}

1. Dimitrakos, T., et al.: Securing Business Operations in an SOA. BT Technology Journal 27(1) (April 2009)

2. Brossard, D., Colombo, M.: Common Capabilities for Trust \& Security in Service Oriented Infrastructures. In: Ferrari, E., Li, N., Bertino, E., Karabulut, Y. (eds.) TM 2009, IFIP AICT, vol. 300, pp. 310-313. Springer, Heidelberg (2009) 